\title{
Entornos virtuales de aprendizaje como apoyo a la enseñanza presencial para potenciar el proceso educativo
}

\section{Virtual learning environments as support for face-to-face teaching to enhance the educational process}

\author{
Rodríguez Andino, Milagro de la Caridad*, Barragán Sánchez, Hilda María \\ Universidad Católica de Cuenca, Sede Macas \\ Macas, 140150, Ecuador \\ *mrodrigueza@ucacue.edu.ec
}

\begin{abstract}
Resumen
La incorporación por parte de profesores y estudiantes de los medios y recursos informáticos al proceso de enseñanza aprendizaje en la educación superior, determina el perfil de la universidad moderna, lo cual, supone un proceso integral que fomente el aprendizaje autónomo e implique la creación de una cultura para la apropiación crítica del conocimiento. Este problema ha llevado a realizar diferentes estudios relacionados con el empleo de Entornos Virtuales soportados por un amplio uso de las TIC para apoyar la enseñanza presencial con lo virtual. Esta investigación se orientó hacia la necesidad de promover la utilización de un (EVE/A) sustentado sobre aspectos teóricos que posibilitan el desarrollo de habilidades, valores, sentimientos y nuevos umbrales de representación cognitiva, que influyen en el aprendizaje de quienes interactúan con estos entornos, convirtiéndolos en un poderoso mediador educativo. Se utilizó una muestra de 22 estudiantes de la carrera de Ingeniería Empresarial de la Sede Macas. Aplicando la observación científica, la revisión de las actividades colocadas en los cursos diseñados en el EVE/A, las entrevistas y encuestas a profesores y estudiantes, se recogieron los datos que se procesaron estadísticamente obteniendo análisis porcentuales, y medias aritméticas para elaborar tablas y gráficos. Como principal resultado se destaca que la aplicación de estrategias dirigidas a la solución de problemas de manera individual y colectiva, así como, el trabajo colaborativo en grupo, basados en una eficaz gestión de contenidos y de información con el EVE/A, permitieron la mejora del rendimiento individual y grupal de los estudiantes potenciando su aprendizaje, la actitud positiva hacia la investigación científica y su aplicación en la vida profesional. La conclusión fundamental indica que con el uso de estas tecnologías es posible implicar más a los estudiantes en su proceso de aprendizaje y mejorar su rendimiento académico desarrollando habilidades para el manejo de estos entornos.
\end{abstract}

Palabras clave: Entornos Virtuales de Aprendizaje, Uso de las TIC, Diseño de Cursos en Ambientes virtuales.

\begin{abstract}
The incorporation by teachers and students of the means and computer resources to the teaching-learning process in higher education, determines the profile of the modern university, which, supposes a comprehensive process that encourages autonomous learning and involves the creation of a Culture for the critical appropriation of knowledge. This problem has led to different studies related to the use of Virtual Environments supported by an extensive use of ICTs to support classroom and virtual teaching. This research was oriented towards the need to promote the use of a $(E V E / A)$ based on theoretical aspects that enable the development of skills, values, feelings and new thresholds of cognitive representation, which influence the learning of those who interact with these environments, Making them a powerful educational mediator. A sample of 22 students from the Business Engineering career at Macas Headquarters was used. Using the scientific observation, the review of the activities placed in the courses designed in the EVE/A, the interviews and surveys to teachers and students, collected the data that were processed statistically obtaining percentage analyzes, and arithmetic averages to draw tables and graphs. The main result is that the application of strategies aimed at problem solving in an individual and collective manner, as well as group collaborative work, based on an effective content and information management with the EVE / A, allowed the improvement Individual and group performance of students, enhancing their learning, positive attitude towards scientific research and their application in professional life. The fundamental conclusion is that with the use of these technologies it is possible to involve students more in their learning process and improve their academic performance by developing skills in the management of these environments.
\end{abstract}

Key words: Virtual Learning Environments, Use of the TIC, Courses design in virtual Environments. 


\section{Introducción}

Con la integración de las tecnologías en los procesos de enseñanza aprendizaje, la educación tiende a desarrollarse como un sistema abierto y permanente que exige la innovación de enfoques pedagógicos modernos para favorecer el estudio autónomo e independiente, el trabajo en equipo, el desenvolvimiento de procesos interactivos de comunicación y apropiación del conocimiento, mediados por la acción dialógica entre profesores y estudiantes, así como por el uso de modernas tecnologías de la información y las comunicaciones.

La incorporación de los medios y recursos informáticos, así como de los métodos activos, al proceso de enseñanza aprendizaje en la educación superior es una necesidad que caracteriza la universidad actual, lo cual supone un proceso integral e integrado de aprendizaje autónomo e implica la creación de una verdadera cultura para la apropiación crítica de la realidad y de sí mismo y para la apropiación del conocimiento.

Las investigaciones consultadas señalan que, en particular, los métodos que garanticen el aprendizaje activo, soportados en el amplio empleo de las TIC y en el diseño de nuevos escenarios de aprendizaje con el uso óptimo de esos recursos, constituyen una premisa indispensable para lograr el pleno acceso y deben estar presentes en todas las modalidades de estudio, además se debe garantizar el control del aprendizaje por parte del propio estudiante.(Cazares, 2010); (Horrutinier, 2009).

En otros estudios realizados por (Pérez, Fernández, y Braojos, 2010); (Belloch, 2010); (Salinas, 2012); (Maza, Andino, Sentí, y Rodriguez, s.f.) se comparten criterios señalando que los entornos virtuales de enseñanzaAprendizaje (EVE/A) facilitan la divulgación de contenidos formativos, favoreciendo la comunicación entre los participantes del proceso y el desarrollo de habilidades, motivos, intereses y la construcción compartida de significados en un ambiente rico en información y en oportunidades para gestionar información y conocimiento, son una herramienta eficaz para potenciar los cambios necesarios y apoyar el desarrollo de nuevos modelos como el presencial ó el modelo semipresencial con un amplio soporte de las tecnologías.

Las investigaciones realizadas en esta temática por la autora principal de este trabajo proporcionaron un conjunto de criterios sobre el papel que desempeñan los profesores en los entornos virtuales, así como las principales dificultades que se presentan, entre las que se mencionan: la falta de preparación de los docentes para: el diseño y desarrollo de cursos virtuales, la gestión de procesos de formación no presenciales, el manejo de entornos tecnológicos y la gestión de información y conocimiento a través de los mismos (Maza y cols., s.f.); (M. R. Andino, Sentí, y Rodríguez, 2008); (M. R. Andino, Sentí, y Rodríguez, 2010); (C. M. R. Andino y Ramírez, 2013); (M. R. Andino, Senti, Rodríguez, Colina, y Maza, 2014).

Entre los principales problemas encontrados a través de la aplicación de encuestas y entrevistas tiene que ver con la falta de conocimiento por parte de los docentes de metodologías y fundamentos para el diseño de los cursos y las limitaciones en el uso de estrategias de aprendizaje más efectivas en estos entornos que influyan en el desarrollo integral de los estudiantes.

El objetivo de esta investigación se orientó hacia la necesidad de promover la utilización de un (EVE/A) sustentado sobre aspectos teóricos que posibiliten el desarrollo de habilidades, valores, sentimientos y nuevos umbrales de representación cognitiva, que influyan en el aprendizaje de quienes interactúan con estos entornos, convirtiéndolos en un poderoso mediador educativo.

En el artículo se se valoran los estudios realizados acerca de los sustentos teóricos para el diseño y desarrollo de los cursos soportados en los entornos virtuales de aprendizaje con la aplicación del trabajo colaborativo en grupo para favorecer el autoaprendizaje, el desarrollo individual y colectivo y el papel activo del estudiante en la formación de habilidades generales relacionadas con la información y la gestión del conocimiento, mediante el uso de herramientas de tecnología educativa de avanzada que permitan dar respuesta a las exigencias de los modelos tanto presencial, como semipresencial, desde este punto de vista se presenta su utilidad en el campo académico y profesional.

La experiencia se desarrolló en el 2 do ciclo de la carrera de Ingeniería Empresarial de la Sede Macas de la Universidad Católica de Cuenca en el período marzoagosto 2015, para valorar si la combinación de las actividades presenciales con las actividades en el entorno virtual favorecen el rendimiento académico de los estudiantes y el desarrollo de habilidades para el manejo de estas tecnologías.

Las limitaciones de la investigación estuvieron dadas por la predisposición que presentaron los estudiantes al principio de la experiencia, para trabajar en el entorno virtual, lo cual requirió un mayor trabajo de las docentes para motivarlos en la realización de tareas individuales y actividades grupales con el uso de estas tecnologías.

\subsection{Marco Referencial}

La incorporación de las TIC al proceso de enseñanza aprendizaje como necesidad formativa de la época y soporte tecnológico de una nueva cultura del aprendizaje debe ser a partir de la asimilación de estas tecnologías por parte del profesor. Para que este logre integrarlas creativamente en un proceso de enseñanza-aprendizaje desarrollador, con modalidad presencial o semipresencial, se requiere que el mismo pueda disponer de determinados elementos teóricos que lo guíen desde el punto de vista didáctico, pedagógico y tecnológico hacía la consecución de ese fin.(Maza y cols., s.f.)

Para tomar decisiones sobre la forma de aplicar los entornos virtuales a la enseñanza presencial o semipresencial es necesario basarse en fundamentos, tantos psicológicos 
como epistemológicos y de principios que se derivan de la concepción que se tenga de aprender y enseñar. A partir de estos se definirán (M. R. Andino y cols., 2008), (M. R. Andino y cols., 2014); (Belloch, 2010):

- La integración de elementos virtuales y actividades presenciales.

- Los criterios para la selección y organización de los contenidos.

- Las actividades de enseñanza y evaluación, su secuencia.

- Los roles de profesores y estudiantes, sus relaciones.

- Los recursos.

En la implementación se necesita el uso de tecnologías que permitan poner en práctica actividades de enseñanza y aprendizaje que potencien la comunicación recíproca, la integración de medios, el uso de materiales y recursos para el aprendizaje y el trabajo colaborativo.

Los entornos virtuales por sus características pueden constituirse como un poderoso mediador en la modalidad presencial y semipresencial.

La autora principal del trabajo considerando al enfoque histórico cultural, como una de las fuentes teóricas consultadas, al cual se incorporan aportes de otras concepciones como el aprendizaje desarrollador, define a un EVE/A como un "Espacio de comunicación que hace posible, la creación de un contexto de enseñanza y aprendizaje en un marco de interacción dinámica, a través de contenidos culturalmente seleccionados y elaborados y actividades interactivas para realizar de manera colaborativa, utilizando diversas herramientas informáticas soportadas por el medio tecnológico, lo que facilita la gestión del conocimiento, la motivación, el interés, el autocontrol y la formación de sentimientos que contribuyen al desarrollo personal".

Porqué usar un espacio virtual de aprendizaje. La introducción de un EVE/A en la educación superior se justifica como señalan (Sentí y Lara, 2010) cuando:

- Se logra un aumento notable en la calidad y cantidad de los materiales y recursos de aprendizaje, propios y ajenos, y se facilita su acceso a los estudiantes.

- Se mantiene una actualización permanente de los materiales y recursos.

- Se facilita el proceso de comunicación entre los profesores y los estudiantes y entre los propios estudiantes, al complementar las actividades cara a cara. Un acercamiento real al aprendizaje colaborativo.

- Se garantiza mayor flexibilidad y variedad de las actividades que forman el núcleo del currículum.

- Se influye en la formación de los estudiantes para crear habilidades para el uso de herramientas tecnológicas y meta cognitivas («aprender a aprender», planificación del propio aprendizaje, auto evaluación, etc.).

- Se flexibiliza el «tiempo de estudio» para acomodarse a los problemas y potencialidades de los estudiantes; etc.

Pero lo anterior no se logra con solo tener una buena intensión y proponer su uso, incluso contando con la última tecnología, disponiendo de banda ancha para la transmisión de información, voz y datos. Es necesario utilizar metodologías, conceptos pedagógicos y didácticas particulares que logren una real relación y comunicación entre el que enseña y el que aprende, entre ellos directamente y entre ambos y las tecnologías.(Sentí y Lara, 2010)

Fundamentos para el diseño e implementación de cursos en entornos virtuales de enseñanza-aprendizaje. La novedad que ofrecen los EVE/A viene dada por la integración de recursos semióticos como el lenguaje oral y escrito, lenguaje audiovisual, gráfico o numérico y las posibilidades de comunicación, intercambio, acceso y procesamiento de la información que ofrecen, lo que puede crear eventualmente un nuevo entorno de aprendizaje en condiciones inéditas para operar la información, transformarla en conocimiento y además desarrollar habilidades, sentimientos y valores que contribuyan al desarrollo personal tanto del estudiante como del docente.

En el campo del diseño educativo de cursos sustentados en EVE/A, la preocupación está en la aplicación de teorías orientadas hacia la promoción de actividades cognitivas e interactivas más potentes, así como a la reorganización y extensión de los aprendizajes en un sentido de construcción activa del conocimiento, vista esta como la individualización de la experiencia social, cultural de manera única e irrepetible por cada sujeto, lo que supone el tránsito de lo externo a lo interno en palabras de (Vigotsky, 1979), de lo interpsicológico a lo intrapsicológico, de la dependencia a la independencia, de la regulación externa a la autorregulación. Así uno de los principales retos de la formación apoyada con las TIC es ofrecer nuevas representaciones y perspectivas de distintos fenómenos, de interés tanto científico como cotidiano, que de otra manera no sería posible desarrollar; y de esta forma contribuir a transformar tanto nuestra comprensión y prácticas como la cultura misma, devolviendo así, en palabras de (Fariñas, 2004), a la cultura en que vive una obra en correspondencia con el patrimonio heredado.

El uso de los EVE/A facilita al estudiante la interacción social con otras personas que pueden representar los agentes mediadores. Esta tecnología que presenta una forma de interacción proveerá el desarrollo de las habilidades interpersonales y eliminará barreras culturales a partir de que estudiantes y profesores se comuniquen a través de las nuevas formas que propone este medio.

$\mathrm{El}$ profesor es un agente cultural y un mediador entre los productos sociohistóricos y los procesos de apropiación de los alumnos (Bermúdez, 2001). Es quien estructura las situaciones de aprendizaje, organiza el proceso de dominio progresivo por parte de los estudiantes de las estrategias y modos de actuar, plantea los retos brinda modelos, sugerencias alternativas, ayuda y guía paulatinamente la ampliación de las zonas de desarrollo potencial y el tránsito del control externo al interno individual.

Explotar los diferentes modelos de comunicación a 
los que se puede recurrir en los EVE/A, en función de los recursos pedagógicos, las actividades que se propongan, o el tipo de materiales por los que se opte (Maza y cols., s.f.), hacen que la comunicación entre el profesor, tutor o experto y el grupo se enriquezca y humanice. Son la actividad y la comunicación las vías que tiene el individuo para expresar lo más íntimo de sí y construir creativamente la obra que dará lugar a su desarrollo y por ende a sí mismo (Fariñas, 2005).

La variedad de interacciones de carácter interpersonal que pueden tener lugar en esta clase de entornos pueden ser: comunicaciones uno-a-uno en las consultas personales al profesor para la resolución de dudas, aclaraciones individuales, etc., y en las comunicaciones entre estudiantes; comunicaciones uno-a-muchos a través de lecturas, enunciados de ejercicios, recomendaciones generales, etc.; y comunicaciones muchos-a-muchos en debates, discusión de casos, simulaciones, tormenta de ideas, etc.(Maza y cols., s.f.)

Esta interacción social lograda a través del EVE/A facilita el desarrollo cognitivo del individuo, premisa que se deriva de la "Zona de Desarrollo Próximo", teoría que Vigotsky define como:"'la distancia entre el nivel real de desarrollo, determinada por la capacidad de resolver independientemente un problema, y el nivel de desarrollo potencial, determinado a través de la resolución de un problema bajo la guía de un adulto o en colaboración con otro compañero más capaz”. (Vigotsky, 1988).

En estos procesos de interacción logrados a través del EVE/A se producirá la construcción de significados compartidos entre el profesor y los estudiantes; entre estudiantes y entre los alumnos y otras personas que pueden no pertenecer a la institución educativa (tutores o expertos del ámbito empresarial).

Es un proceso intrínsecamente mediado por otras personas, al mismo tiempo constructivo, cultural y comunicativo (Coll, Mauri, y Onrubia, 2008). Un proceso, en definitiva, de carácter personal pero no individual.

Vigotsky considera el aprendizaje como una actividad social de producción y reproducción del conocimiento. Pone en el centro de la atención al sujeto activo, consciente, orientado hacia el objetivo; en interacción con otros sujetos (el profesor, el tutor, el asesor y otros estudiantes); sus acciones con el objeto con la utilización de diversos medios en condiciones socio-históricas determinadas. Partiendo de la idea de Vigotsky de que un rasgo fundamental de la actividad transformadora del hombre es su carácter mediatizado por el instrumento que se interpone entre el sujeto y el objeto de la actividad; lo central en el proceso de enseñanza aprendizaje a través de los EVE/A consistirá en estudiar las posibilidades de asegurar las condiciones (materiales interactivos, sistema de relaciones, tipos de actividad, funciones de la comunicación) para que el estudiante se eleve mediante la actividad colaborativa a un nivel superior.

En la didáctica actual con una concepción desarrolladora, aunque el centro del aprendizaje es el sujeto que aprende, el aprendizaje es un proceso de participación, de colaboración y de interacción. En el grupo, en la comunicación con los otros, las personas desarrollan el autoconocimiento, compromiso y la responsabilidad individual y social, elevan su capacidad para reflexionar divergente y creadoramente, para solucionar problemas y tomar decisiones (Castellanos, 2002). En este sentido el EVE/A facilita el papel protagónico y activo del sujeto que aprende y favorece la mediación social tanto del profesor como del tutor o el experto a través de las herramientas de gestión de recursos y de las posibilidades de comunicación sincrónica y asincrónica que el mismo ofrece. (M. R. Andino y cols., 2010).

El aprendizaje esta mediado por la existencia de otros (el profesor, el grupo, la cultura expresada en el currículo y los materiales educativos) y de la actividad de comunicación que constituye una característica esencial de este proceso y que ahora con el uso de los EVE/A se ve favorecida y convertida en un proceso sujeto-sujeto y sujeto consigo mismo, donde el elemento fundamental es el diálogo que se establece mediante las herramientas que brinda este tipo de entorno y el intercambio bidireccional de información, conocimientos, motivos, intereses y sentimientos.

Considerando esta concepción de aprendizaje y del carácter principal de la enseñanza en el desarrollo del alumno el proceso de enseñanza-aprendizaje a través de los EVE/A deben considerarse diferentes aspectos metodológicos. Existen principios establecidos por varios autores (Sentí, Rodríguez, Andino, y Tarke, 2011) que no siempre son tenidos en cuenta por investigadores de tecnología educativa que convierten en inconsistente muchas de las propuestas realizadas. Cuatro de estos principios (Sentí y Lara, 2010), se formulan aproximadamente de la siguiente manera:

1) Formulación de los objetivos o propósitos a lograr a partir de las acciones que debe desarrollar el estudiante en el marco de las materias específicas y de las funciones que éstas desempeñan en el perfil del egresado (Sanz, 1999).

2) Formulación de problemas-tareas-actividades de aprendizaje con valor sociocultural - personal real para orientar la búsqueda de la información y del conocimiento necesario e ir en aras de su resolución y propiciar en los estudiantes, el desarrollo de habilidades y el sentido personal-social y constructivo de sus tareas de aprendizaje (Fariñas, 2004).

3) Formular tareas y actividades de aprendizaje que utilizando las novedades tecnológicas de las herramientas de gestión de contenidos, dentro del entorno virtual de aprendizaje y fuera de el, procurando que propicien la capacidad para problematizar el conocimiento y la búsqueda de las regularidades de los fenómenos y procesos implicados en las tareas, activando conscientemente el aprendizaje y por ende el desarrollo (Fariñas, 2004).

4) La búsqueda del desarrollo de puntos de vista en el educando, sobre la realidad y actitudes congruentes con 
estos, como consecuencia de la realización de las tareas de aprendizaje y la organización de los conocimientos a través de las herramientas del entorno virtual, para resolverlos (Fariñas, 2004).

Los EVE/A al generar nuevos contextos posibilitan el desarrollo de habilidades, valores, sentimientos y nuevos umbrales de representación cognitiva, que influyen en las oportunidades de aprendizaje de quienes interactúan con estos, convirtiendo a los EVE/A en un poderoso mediador educativo.

En un EVE/A no solo se actúa con el mismo en el proceso de formación, sino que paralelamente se ejecuta la inteligencia, la creatividad y los sentimientos y con todo esto, las estrategias para aprender a aprender lo que implica según (Fariñas, 2005) toma de conciencia de sí mismo, de las actitudes hacia el conocimiento y de las actitudes hacia los demás, saber participar en grupo y cooperar, saber obrar el conocimiento para su interiorización personalizada. En un proceso de este tipo todos aportan algún saber y todos pueden enseñar y aprender algo, por lo que siempre son sujetos y nunca objetos del proceso.

\section{Metodología}

La investigación desarrollada tuvo un enfoque cuanticualitativo, con un alcance descriptivo-explicativo, en el que se aplicaron métodos como el histórico - lógico para realizar un estudio que permitiera conocer la esencia del problema relacionado con el empleo del (EVE/A), la tendencia de esa problemática y el estado actual en el campo internacional. Se utilizó además la observación para valorar la situación relacionada con la utilización del EVE/A y la creación de los cursos, recopilando la información acerca de su comportamiento.

La experiencia se desarrolló con 22 estudiantes del 2do ciclo de la carrera de Ingeniería Empresarial de la Sede Macas de la Universidad Católica de Cuenca, en el período de marzo 2015 a agosto 2015, en la asignatura de Metodología de la Investigación.

En este período se utilizaron técnicas de aprendizaje cooperativo, para alcanzar la interacción y la comunicación en la asignatura y potenciar el autoaprendizaje, el desarrollo individual y colectivo y el papel activo del estudiante en su propio proceso de formación, desarrollando habilidades generales relacionadas con la información y la gestión del conocimiento, mediante el uso de herramientas de tecnología educativa de avanzada.

El entorno virtual se convirtió en un espacio de trabajo, de cooperación y comunicación donde el alumnado expresó sus comentarios y dudas. En él se colocó el material producido por el grupo para que fuera consultado por todos, tanto sobre la realización de las tareas relacionadas con el proyecto de investigación que debían desarrollar, como sobre los conceptos teóricos trabajados.

Se aplicaron instrumentos cualitativos (observación y entrevistas) y cuantitativos (encuestas) para la recogida de datos. Las encuestas y entrevistas se utilizaron para conocer el estado de opinión de profesores y estudiantes sobre el uso del EVE/A y el diseño de los cursos en la carrera.

Finalmente se realizó el procesamiento estadístico de los datos con la aplicación Excel, obteniendo análisis porcentuales, y medias aritméticas para elaborar tablas y gráficos que permitieron realizar una valoración de los resultados obtenidos con la aplicación de los instrumentos.

\section{Resultados}

En el diagnóstico efectuado en la investigación desarrollada, se observaron y revisaron los diseños de los cursos que estaban implementados en la Plataforma utilizada, las autoras se percataron que no existe una homogeneidad de criterios en cuanto al modelo para el diseño, así como tampoco se utilizan las estrategias de aprendizaje más adecuadas a estos entornos, por tal razón se investigó sobre los diferentes métodos del proceso de diseño de los cursos virtuales para transformar de manera paulatina el modelo de enseñanza tradicional presencial en el pregrado hacia un modelo donde se aumenten las actividades participativas más directamente vinculadas a la formación personal y a la apropiación de aprendizajes que preparen a los estudiantes para el desarrollo de habilidades, motivos, intereses, sentimientos y para la gestión de información y conocimiento relacionadas con la investigación científica.

\subsection{Resultados de la Aplicación en la Práctica}

A continuación se muestra las experiencias obtenidas al poner en práctica el curso elaborado a partir de la utilización del aprendizaje cooperativo y sustentado en los fundamentos teóricos expuestos. En la experiencia participaron las autoras de este trabajo como profesoras de la asignatura metodología de la investigación.

La Plataforma Interactiva para la implementación del curso fue MOODLE.

En el curso diseñado en la asignatura relacionada con la formación investigativa se han incluido un conjunto de módulos de actividades de aprendizaje cooperativo e interactivo. Los trabajos de los estudiantes mediante estas actividades fueron enviados y calificados por los profesores a través de los módulos de Tareas o Talleres.

También se incluyeron actividades para calificar de forma automática mediante los Cuestionarios, los cuales constituyen una herramienta potente y flexible para evaluar ciertos conocimientos de los alumnos y se añadieron ejercicios sobre la elaboración del proyecto de investigación.

La comunicación en el curso se realizó a través de los Foros para debates y el correo para el intercambio de mensajes relacionados con el curso. Se trabaja de forma colaborativa mediante los Wikis y los Blogs.

El contenido se presentó y gestionó usando las actividades de Lecciones y SCORM. Las palabras claves del curso se agregarón en los Glosarios.

Las Encuestas y las Bases de Datos son actividades de gran ayuda, se utilizaron para el control de trabajos 
de la asignatura o seguimiento y control de actividades evaluativas.

Este panorama permitió centrarse en algunas de las actividades que se han abarcado para iniciar un trabajo que aún se esta perfeccionando y del cual se va en busca de mayor dominio:

- Las lecciones son un cambio de ritmo interesante para los alumnos y ofrecen más información e interacción que en un texto normal con más preparación por parte del profesor que otro tipo de herramientas.

- La realización de tareas se combinan con el recurso páginas Web haciendo enlaces hacía sitios con información. Se incluyeron imágenes auxiliares para visualmente apoyar la tarea a cumplir.

Para evaluar los resultados del curso se definieron las siguientes variables de estudio:

1) Mensajes recibidos por el profesor a través del correo electrónico.

2) Cantidad de visitas de los estudiantes a los recursos y materiales.

3) Tareas enviadas en tiempo.

4) Participación en las actividades de trabajo colaborativo.

5) Resultados del rendimiento en las evaluaciones parciales efectuadas de manera presencial.

Los datos fueron recogidos en la tabla 1, registrando el valor numérico correspondiente a cada variable por estudiante:

Tabla 1

Formato para registrar los datos

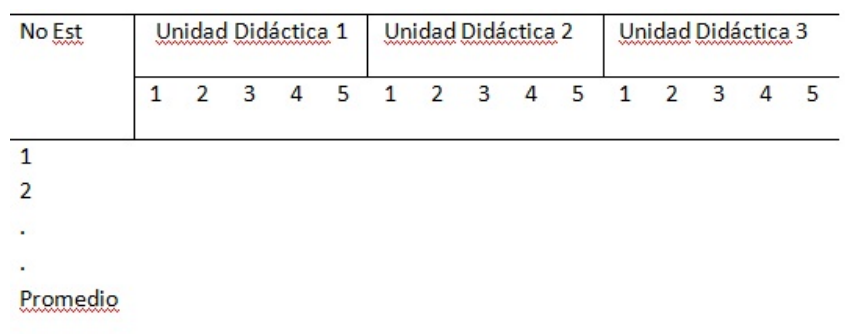

Fuente: Datos recogidos del análisis del curso por cada variable observada

Una vez procesados los datos en la aplicación Excel se obtuvo el gráfico Fig. 1 con la representación de los promedios obtenidos por cada una de las variables pudiéndose observar la evolución obtenida por cada una de ellas en el tránsito de la 1ra unidad de estudio en el sílabo de la asignatura hasta la tercera unidad.

Un análisis de estos resultados evidencia que al comienzo del desarrollo de la asignatura utilizando el curso sustentado en el EVE/A los estudiantes no tenían el hábito de revisar los materiales ( 2.17 prom en la 1ra UD), ni de enviar las tareas utilizando el entorno virtual ( 0.65 prom en la 1ra UD), mucho menos de participar en las actividades

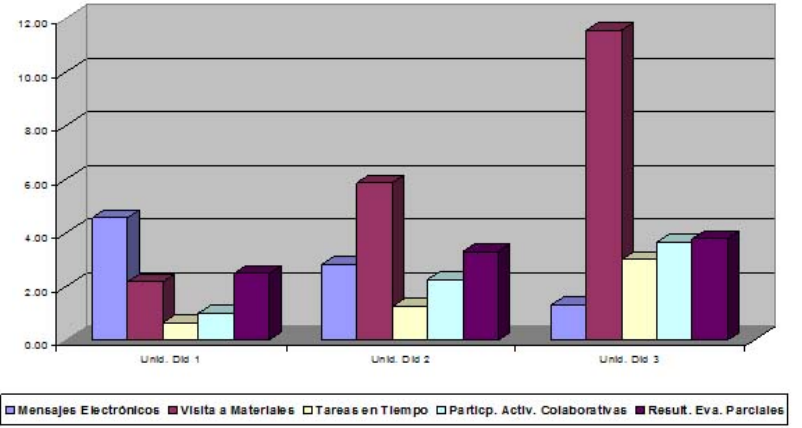

Figura 1. Muestra del promedio de las variables de estudio por cada Unidad Didáctica en el curso de la Asignatura.

Fuente: Procesamiento de los resultados del análisis de las variables.

colaborativas (1.00 prom en la 1ra UD) para el análisis de las preguntas, discusión de los ejemplos o solución de los problemas que fueron indicados en cada unidad según las estrategias de aprendizaje apropiadas para el tratamiento del contenido.

Inicialmente los estudiantes no accedían a los Foros, ni enviaban las tareas en tiempo en su mayor parte, pero luego motivados con el uso de herramientas de comunicación (Chat) y Blogs se incremento la participación en las actividades colaborativas.

Por las intervenciones que realizaban los estudiantes en las actividades presenciales las profesoras tuvimos la impresión que la causa fundamental podía estar en la falta de autonomía de los alumnos para realizar las actividades de autoaprendizaje a través del entorno.

Esta impresión fue corroborada a través del análisis de los mensajes electrónicos recibidos. Para estudiar los mensajes estos se clasificaron en cuatro grupos, mensajes vinculados al contenido y estructura del curso, mensajes que resaltan problemas, mensajes relacionados con trabajos a enviar, mensajes varios (sin relación directa con el curso). La distribución de los 200 mensajes recibidos se muestra en la Fig. 2.

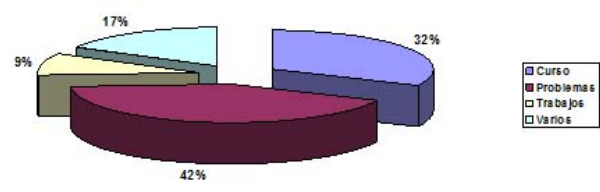

Figura 2. Gráfica de la distribución porcentual de los diferentes mensajes electrónicos recibidos

Fuente: Procesamiento de los resultados del análisis de las variables.

Los mensajes electrónicos incluidos en la categoría "Problemas" fueron clasificados en tres tipos: técnicos, de autonomía y otros. Un estudio de estos mensajes permitió observar que los mensajes relacionados con los problemas técnicos pasaron de un 34 porciento en la 1ra UD a un 1 
porciento en la última, mientras que los relacionados con la autonomía pasaron de un $49 \%$ a un $3 \%$.

Los problemas técnicos indican que al principio los estudiantes necesitan un tiempo para acostumbrarse al entorno virtual y desarrollar las habilidades para interactuar con el mismo.

El análisis de las transcripciones de los correos electrónicos recibidos parece subrayar la sobrecarga cognitiva generada por una situación demandante de autonomía y de responsabilidad, en un nivel superior a lo habitual para los estudiantes. No estando acostumbrados a tal demanda, su reacción inicial, en relación con sus actitudes y motivación frente a este nuevo ambiente de aprendizaje, fue negativa. Este resultado queda manifiesto cuando se pone atención particular a los problemas experimentados por los estudiantes y a los resultados de las evaluaciones efectuadas presencialmente.

En efecto, la disposición de los estudiantes fue mucho más elevada al final que al principio del curso, siendo necesario un esfuerzo inicial evidente para aclimatarse a esta nueva forma de recibir la docencia, donde están obligados a desarrollar un papel más activo. Los profesores necesitamos orientar a los estudiantes para que piensen de forma diferente y se apropien de una nueva manera de aprender. Este trabajo es muy importante realizarlo desde los primeros años de la carrera.

Esta nueva forma de aprender parece haber imbuido a los alumnos de una mayor motivación.

Además, incluso si varios estudiantes no tenían la costumbre de autoaprender y de trabajar colaborativamente de acuerdo a las estrategias de aprendizaje diseñadas, son varios aquellos que la indicaron como un logro del curso. Otros incluso afirmaron que "el hecho de tener una responsabilidad mayor les empujaba a trabajar con más ahínco, a participar más activamente, permitiéndoles aprender a aprender"(estudiante).

En realidad, bien parece posible que el cambio de disposición de los estudiantes frente a este modo de aprendizaje, reflejado inicialmente por su falta de responsabilidad y de autonomía en relación con sus propios aprendizajes, no es consecuencia directa de la exposición al modo de aprendizaje utilizando un EVE/A. Puede ser, más bien, el resultado de la dimensión de madurez o de experiencia de la inhabitual autonomía, ya que el estudiante no esta acostumbrado a esta autonomía en el modelo "tradicional", en el cual él es condicionado a depender, en sus conductas, del docente o de cualquiera forma de control externo.

Los comentarios en la encuesta y la entrevista relacionados con la interacción estudiante-profesor fueron en general favorables (con excepción de un estudiante calificado como de bajo rendimiento, quien no aprendió a utilizar los recursos del entorno virtual) y hacen suponer que se logró un buen grado de presencia social a través del EVE/A; las profesoras percibimos una excelente comunicación con los alumnos, con mayor grado de cercanía que en el aula tradicional. A pesar de ser una de sus primera experiencia en un curso que combina lo presencial con lo virtual se logró la participación de la mayoría de los estudiantes, sobre todo en la última unidad didáctica, al parecer, influyeron las respuestas oportunas a las preguntas que se le enviaban y al tono amigable e informal que se le daba a sus aportaciones en las discusiones diferidas y a sus mensajes en el correo electrónico.

Por las opiniones expresadas, se puede decir que la interacción estudiante-entorno virtual fue muy eficiente. (Guanawardena, 1995) menciona que la interacción que se da entre el estudiante y la tecnología es un componente crítico que normalmente ha estado ausente en la literatura y que incluye el conocimiento y uso de la interfaz para todas las interacciones, ya que los estudiantes tienen que realizar un cambio de conducta al tener que utilizar este tipo de dispositivos. El grado de satisfacción en cuanto a la funcionalidad de la estructura del curso e interfaz del EVE/A fue muy alto; no se encontró ningún comentario negativo o contrario a la facilidad de "navegar" dentro del curso y encontrar la información.

Finalmente se pudo constatar que los estudiantes mejoraron tanto el rendimiento individual como grupal potenciando el aprendizaje, su actitud positiva hacia la investigación científica y su aplicación en la vida profesional, además de potenciar la creatividad y la gestión del conocimiento para la solución de problemas en el seno de su grupo virtual.

\section{Conclusiones}

El análisis realizado plantea la importancia de una gestión didáctica y pedagógica rigurosa al diseñar los cursos virtuales, en otras palabras, la necesidad de crear cursos que hagan uso de la tecnología para favorecer el aprendizaje, al servicio del proceso docente y ello con el propósito de acrecentar la motivación de los estudiantes, al mismo tiempo que ayudarlos a adquirir autonomía en su propio proceso de aprendizaje, en el que juega un papel importante las estrategias de aprendizaje que sean orientadas por el profesor y utilizadas por los alumnos, así como el trabajo colaborativo en grupo y las interacciones que se logren entre el estudiante y el EVE/A, estudiante-contenido, estudiante-profesor y estudiante-estudiante, cuestiones que están recogidas en los sustentos señalados que sirven como soporte teórico al diseño de los cursos de las asignatura objeto de la experiencia.

La cantidad de trabajo requerido para obtener la interactividad puede variar según los conocimientos previos y la experiencia con el entorno virtual, trabajar con Moodle no se considera como el resultado de un producto, es un proceso activo de aprendizaje y lleva a los profesores a reflexionar sobre su propia práctica.

Con el uso de los entornos virtuales es posible implicar más a los estudiantes en su proceso de aprendizaje y mejorar su rendimiento académico y desarrollar habilidades para el manejo de estos entornos. 


\section{Referencias}

Andino, C. M. R., y Ramírez, C. E. F. M. (2013). La gestión de información y la infotecnología en la formación del profesional de las ciencias económicas y empresariales. Retos de la Dirección, 5(1).

Andino, M. R., Sentí, V. E., y Rodríguez, J. F. (2008). Una estrategia para el diseño e implementación de cursos virtuales de apoyo a la enseñanza semipresencial en la carrera de economía de la universidad de camagüey (Tesis Doctoral no publicada). Universidad de la Habana.

Andino, M. R., Sentí, V. E., y Rodríguez, J. F. (2010). Empleo de entornos virtuales de aprendizaje para el apoyo a la enseñanza presencial y semipresencial.

Andino, M. R., Senti, V. E., Rodríguez, J. F., Colina, F. G., y Maza, J. C. (2014). Gestión de la educación virtual para la formación continua de profesionales de perfil empresarial. Gestión en el Tercer Milenio, 8(16), 93 103.

Belloch, C. (2010). Entornos virtuales de aprendizaje. Unidad de tecnología Educativa.

Bermúdez. (2001). La teoría histórico cultural [Manual de software informático]. Facultad de Psicología..

Castellanos, y. O., Castellanos. (2002). Aprender y enseñar en la escuela. una concepción desarrolladora. La Habana: Editorial Pueblo y Educación.

Cazares, S. I. (2010). Entornos virtuales de aprendizaje. Investigación, 15(45), 423-452.

Coll, C., Mauri, T., y Onrubia, J. (2008). El análisis de los procesos de enseñanza y aprendizaje mediados por las tic: una perspectiva constructivista. E. Barberà, T. Mauri, y J. Onrubia (Coords.), Cómo valorar la calidad de la enseñanza basada en las TIC, 47-60.

Fariñas. (2004). Psicología educativa. En (cap. La educación del futuro vista desde una psicología históricoculturalista). La Habana: Editorial Pueblo y Educación.

Fariñas. (2005). Psicología educación y sociedad. un estudio sobre el desarrollo humano. La Habana: Editorial Felix Varela.

Guanawardena. (1995). Cuadernos de educación a distancia 3: Enfoques sobre evaluación de los aprendizajes en educación a distancia. En (caps. Nuevos caminos en el aprendizaje, nuevas formas de evaluar). México: Universidad de Guadalajara.

Horrutinier, S. (2009). El modelo de formación. la universidad cubana. Editorial Universitaria.

Maza, J. V. C., Andino, M. R., Sentí, V. E., y Rodriguez, J. F. (s.f.). El aprendizaje basado en problemas y el trabajo colaborativo como fundamentos para el diseño de cursos virtuales. ejemplificación en la asignatura de informática ii para las carreras de perfil empresarial. Gestión en el Tercer Milenio, 12(24), 71-80.

Pérez, H. S., Fernández, S. R., y Braojos, C. G. (2010). Metodologías que optimizan la comunicación en en- tornos de aprendizaje virtual. Comunicar: Revista científica iberoamericana de comunicación y educación(34), 163-171.

Salinas, J. (2012). Reseña del libro diseño y moderación de entornos virtuales de aprendizaje (eva). Revista de Universidad y Sociedad del Conocimiento (RUSC). Vol. 9, n. ${ }^{\circ}$ 1., UOC.

Sanz, y. R. (1999). Tendencias pedagógicas contemporáneas. En (cap. El enfoque Histórico Cultural: $\mathrm{Su}$ contribución a una concepción pedagógica contemporánea). La Habana: Universidad de la Habana, CEPES.

Sentí, V. E., y Lara, Y. (2010). El aprendizaje virtual y la gestión del conocimiento.

Sentí, V. E., Rodríguez, J. P. F., Andino, M. R., y Tarke, A. R. (2011). Los espacios virtuales de aprendizaje y la enseñanza semipresente ya distancia. aspectos metodológicos. Educación Superior, 20.

Vigotsky. (1979). El desarrollo de los procesos psicológicos superiores. Critica.

Vigotsky. (1988). Selección de lecturas de psicología pedagógica y de las edades. En Universidad (Ed.), (Vol. Vol. Tomo III, cap. Interacción entre enseñanza y desarrollo). La Habana.

Recibido: 13 de enero de 2017

Aceptado: 24 de julio de 2017

Rodríguez Andino, Milagro: es PhD. en Ciencias de la Educación, desarrolla investigaciones en el uso de las tecnologías para la educación, en la formación de docentes y en la gestión de información y conocimiento con el uso de las TICs. Es docente de la Carrera de Educación y Coordinadora del Departamento de Investigación en la Sede Macas de la UCACUE

Barragan Sánchez, Hilda: Es Master en Administración y Marketing por la Universidad Tecnológica Indoamericana. Docente de la carrera de Ingeniería Empresarial, Sede Macas, UCACUE.

(Correo electrónico: hbarragans@ucacue.edu.ec) 